\title{
Some new estimations for the upper and lower bounds for the spectral radius of nonnegative matrices
}

\author{
Zheng-ge Huang*, Zhong Xu and Quan Lu
}

\section{"Correspondence:}

ZhenggeHuang@mail.nwpu.edu.cn Department of Applied

Mathematics, Northwestern

Polytechnical University, Xi'an,

Shaanxi 710072, P.R. China

\begin{abstract}
In this paper, some new upper bounds on the spectral radius of the Hadamard product of two nonnegative matrices and some upper and lower bounds on the spectral radius of the iterative matrix of a nonsingular $M$-matrix are given. These bounds improved the corresponding results of Cheng et al. (Appl. Math. E-Notes 5:202-209, 2005), Guo et al. (J. Inequal. Appl. 2013:433, 2013), Huang (Linear Algebra Appl. 428:1551-1559, 2008) and Liu et al. (Linear Algebra Appl. 432:936-948, 2010) and are sharper than the related results of Fang (Linear Algebra Appl. 425:7-15, 2007) and Liu and Chen (Linear Algebra Appl. 431:974-984, 2009) in some cases. Some numerical examples are used to show the advantages of the results in this paper.
\end{abstract} MSC: $15 \mathrm{~A} 42 ; 15 \mathrm{~B} 34$

Keywords: nonnegative matrix; Hadamard product; nonsingular M-matrix; spectral radius

\section{Introduction}

For a positive integer $n, N$ denotes the set $\{1,2, \ldots, n\}$. The set of all $n \times n$ complex matrices is denoted by $\mathbb{C}^{n \times n}$ and $\mathbb{R}^{n \times n}$ denotes the set of all $n \times n$ real matrices throughout.

Let $A=\left(a_{i j}\right)$ and $B=\left(b_{i j}\right)$ be two real $n \times n$ matrices. Then $A \geq B(>B)$ if $a_{i j} \geq b_{i j}\left(>b_{i j}\right)$ for all $1 \leq i \leq n, 1 \leq j \leq n$. If $O$ is the null matrix and $A \geq O(>O)$, we say that $A$ is a nonnegative (positive) matrix. The spectral radius of $A$ is denoted by $\rho(A)$. If $A$ is a nonnegative matrix, the Perron-Frobenius theorem (see [1]) guarantees that $\rho(A) \in \sigma(A)$, where $\sigma(A)$ is the set of all eigenvalues of $A$.

For $n \geq 2$, an $n \times n$ matrix $A \in C$ is reducible if there exists an $n \times n$ permutation matrix $P$ such that

$$
P^{T} A P=\left(\begin{array}{ll}
B & C \\
0 & D
\end{array}\right),
$$

where $B$ and $D$ are square matrices of order at least one. If no such permutation matrix exists, then $A$ is irreducible. If $A$ is a $1 \times 1$ complex matrix, then $A$ is irreducible if its single entry is nonzero and reducible otherwise.

Let $A$ be an irreducible nonnegative matrix. It is well known that there exists a positive vector $u$ such that $A u=\rho(A) u, u$ being called the right Perron eigenvector of $A$.

(c) 2015 Huang et al.; licensee Springer. This is an Open Access article distributed under the terms of the Creative Commons Attribution License (http://creativecommons.org/licenses/by/4.0), which permits unrestricted use, distribution, and reproduction in any medium, provided the original work is properly credited. 
Denote by $Z_{n}$ the set of $n \times n$ real matrices all of whose off-diagonal entries are nonpositive. A matrix $A$ is called a nonsingular $M$-matrix (see [1]) if $A \in \mathrm{Z}_{n}$ and the inverse of $A$, denoted by $A^{-1}$, is nonnegative. $A$ is called an inverse $M$-matrix (see [1]) if $A$ is nonsingular and $A^{-1}$ is a nonsingular $M$-matrix. If $A$ is a nonsingular $M$-matrix, then there exists a positive eigenvalue of $A$ equal to $\tau(A)=\left[\rho\left(A^{-1}\right)\right]^{-1}$, where $\rho\left(A^{-1}\right)$ is the spectral radius of the nonnegative matrix $A^{-1} . \tau(A)=\min \{|\lambda|: \lambda \in \sigma(A)\}$ is called the minimum eigenvalue of $A$ (see [2]). The Perron-Frobenius theorem tells us that $\tau(A)$ is an eigenvalue of $A$ corresponding to a nonnegative eigenvector $x=\left(x_{1}, x_{2}, \ldots, x_{n}\right)^{T}$. If, in addition, $A$ is irreducible, then $\tau(A)$ is simple and $x>0$. If $D$ is the diagonal matrix of $A$ and $C=D-A$, then the spectral radius of the Jacobi iterative matrix $J_{A}=D^{-1} C$ of $A$ is denoted by $\rho\left(J_{A}\right)$ (see [3]).

For two matrices $A=\left(a_{i j}\right)$ and $B=\left(b_{i j}\right) \in \mathbb{C}^{m \times n}$, the Hadamard product of $A$ and $B$ is the matrices $A \circ B=\left(a_{i j} b_{i j}\right) \in \mathbb{C}^{m \times n}$. If $A \geq O$ and $B$ is a nonsingular $M$-matrix, then it is clear that $A \circ B^{-1} \geq O$ (see [1]).

Let $\zeta(A)$ represent the set of all simple circuits in the digraph $\Gamma(A)$ of $A$. Recall that a circuit of length $k$ in $\Gamma(A)$ is an ordered sequence $\gamma=\left(i_{1}, i_{2}, \ldots, i_{k+1}\right)$, where $i_{1}, i_{2}, \ldots, i_{k} \in N$ are all distinct, $i_{1}=i_{k+1}$. The set $\left\{i_{1}, i_{2}, \ldots, i_{k}\right\}$ is called the support of $\gamma$ and is denoted by $\bar{\gamma}$. The length of the circuit is denoted by $|\gamma|$ (see [4]).

For convenience, we employ the following notations throughout. Let $A=\left(a_{i j}\right) \in \mathbb{C}^{n \times n}$, we denote, for any $i, j \in N$,

$$
\begin{aligned}
& R_{i}(A)=R_{i}=\sum_{j=1, j \neq i}^{n}\left|a_{i j}\right|, \quad C_{i}(A)=C_{i}=\sum_{j=1, j \neq i}^{n}\left|a_{j i}\right|, \quad R_{i}^{\prime}(A)=R_{i}^{\prime}=\sum_{j=1}^{n} a_{i j} \\
& \sigma_{i}(A)=\sigma_{i}=\frac{R_{i}}{\left|a_{i i}\right|}, \quad \delta_{i}(A)=\delta_{i}=\frac{C_{i}}{\left|a_{i i}\right|} .
\end{aligned}
$$

Recall that $A=\left(a_{i j}\right) \in \mathbb{C}^{n \times n}$ is called diagonally dominant by rows (by columns) if $\sigma_{i} \leq 1$ $\left(\delta_{i} \leq 1\right.$, respectively) for all $i \in N$. If $\sigma_{i}<1\left(\delta_{i}<1\right)$ for all $i \in N$, we say that $A$ is a strictly diagonally dominant by rows (by columns, respectively) (see[1]).

\section{Preliminaries}

Lemma 2.1 [1] Let $A=\left(a_{i j}\right) \in \mathbb{R}^{n \times n}$ be an inverse $M$-matrix, then $A \geq O$.

Lemma 2.2 [5] Let $A=\left(a_{i j}\right) \in \mathbb{C}^{n \times n}$, then

(i) If $A$ is a strictly diagonally dominant matrix by rows, then $A^{-1}=\left(v_{i j}\right)_{n \times n}$ exists, and

$$
\left|v_{j i}\right| \leq \sigma_{j}\left|v_{i i}\right| \quad \text { for all } i \in N
$$

(ii) If $A$ is a strictly diagonally dominant matrix by columns, then $A^{-1}=\left(v_{i j}\right)_{n \times n}$ exists, and

$$
\left|v_{i j}\right| \leq \delta_{j}\left|v_{i i}\right| \quad \text { for all } i \in N \text {. }
$$

Lemma 2.3 [6] Let $A=\left(a_{i j}\right) \in \mathbb{R}^{n \times n}$ be an inverse $M$-matrix, let $A^{-1}=\left(v_{i j}\right)_{n \times n}$, then

$$
v_{i i}>\tau\left(A^{-1}\right)>0 \quad \text { for all } i \in N \text {. }
$$


Lemma 2.4 [7] Let $A=\left(a_{i j}\right)_{n \times n}$ be a nonnegative matrix, then

$$
\rho(A) \leq \frac{1}{2} \max _{i \neq j}\left\{a_{i i}+a_{j j}+\left[\left(a_{i i}+a_{j j}\right)^{2}+4 R_{i} R_{j}\right]^{\frac{1}{2}}\right\} .
$$

Lemma 2.5 [4] Let $A=\left(a_{i j}\right)_{n \times n}$ be a nonnegative matrix, and let $\zeta(A) \neq \phi$. Then for any diagonal matrix $D \in \mathbb{R}^{n \times n}$ with positive diagonal entries, we have

$$
\min _{\gamma \in \zeta(A)}\left[\prod_{i \in \bar{\gamma}} R_{i}^{\prime}\left(D^{-1} A D\right)\right]^{\frac{1}{|\gamma|}} \leq \rho(A) \leq \max _{\gamma \in \zeta(A)}\left[\prod_{i \in \bar{\gamma}} R_{i}^{\prime}\left(D^{-1} A D\right)\right]^{\frac{1}{|\gamma|}} .
$$

Lemma 2.6 [1] Let $A \in Z_{n}, A$ be a nonsingular M-matrix if and only if all its leading principal minors are positive.

Lemma 2.7 [8] Let $A=\left(a_{i j}\right) \in \mathbb{C}^{n \times n}$ and $x_{1}, x_{2}, \ldots, x_{n}$ be positive real numbers. Then all the eigenvalues of $A$ lie in the region

$$
\bigcup_{i=1}\left\{z \in C:\left|z-a_{i i}\right| \leq x_{i} \sum_{j=1, j \neq i}^{n} \frac{\left|a_{j i}\right|}{x_{j}}\right\} .
$$

Lemma 2.8 [9] Let $A=\left(a_{i j}\right) \in \mathbb{R}^{n \times n}$ be a nonsingular M-matrix, then $0 \leq \tau(A) \leq a_{i i}$ for all $i \in N$.

\section{Some upper bounds for the spectral radius of the Hadamard product of nonnegative matrices}

In this section, we give some new upper bounds for $\rho(A \circ B)$, where $A$ and $B$ are nonnegative matrices.

In 2005, Cheng et al. [6] obtained the following result:

Let $A=\left(a_{i j}\right) \in \mathbb{R}^{n \times n}$ be a nonnegative matrix, and let $B$ be an inverse $M$-matrix, $B^{-1}=$ $\left(w_{i j}\right)_{n \times n}$, then

(1) If $A$ is nilpotent, i.e., $\rho(A)=0$, then $\rho(A \circ B)=0$.

(2) If $A$ is not nilpotent, then

$$
\rho(A \circ B) \leq \frac{\rho(A)}{\rho(B)} \max _{1 \leq i \leq n}\left[\left(\frac{a_{i i}}{\rho(A)}+w_{i i} \rho(B)-1\right) \frac{b_{i i}}{w_{i i}}\right] \leq \rho(A) \max _{1 \leq i \leq n} b_{i i} .
$$

Next, we present a new upper bound on $\rho(A \circ B)$, which improves the result in [6].

Theorem 3.1 Let $A=\left(a_{i j}\right) \in \mathbb{R}^{n \times n}$ be a nonnegative matrix, and let $B$ be an inverse $M$ matrix, $B^{-1}=\left(w_{i j}\right)_{n \times n}$. Then

$$
\begin{aligned}
\rho(A \circ B) \leq & \max _{i \neq j} \frac{1}{2}\left\{a_{i i} b_{i i}+a_{i j} b_{j j}+\left[\left(a_{i i} b_{i i}-a_{i j} b_{j j}\right)^{2}\right.\right. \\
& \left.\left.+4 \frac{b_{i i} b_{i j}}{w_{i i} w_{j j}}\left[\rho(A)-a_{i i}\right]\left[\rho(A)-a_{j j}\right]\left[w_{i i}-\frac{1}{\rho(B)}\right]\left[w_{i j}-\frac{1}{\rho(B)}\right]\right]^{\frac{1}{2}}\right\} .
\end{aligned}
$$


Proof Case 1: If $A \circ B$ is irreducible, then $A$ and $B$ are irreducible, since $B$ is an inverse $M$-matrix, by Lemma 2.1 we know $B \geq O$, then by the Perron-Frobenius theorem, there exist two positive vectors $u=\left(u_{1}, u_{2}, \ldots, u_{n}\right)^{T}$ and $v=\left(v_{1}, v_{2}, \ldots, v_{n}\right)^{T}$ such that

$$
A u=\rho(A) u, \quad B^{T} v=\rho\left(B^{T}\right) v
$$

i.e.,

$$
a_{i i}+\sum_{j=1, j \neq i}^{n} \frac{a_{i j} u_{j}}{u_{i}}=\rho(A), \quad\left(B^{-1}\right)^{T} \nu=\frac{1}{\rho\left(\left(\left(B^{-1}\right)^{T}\right)^{-1}\right)} v=\tau\left(\left(B^{-1}\right)^{T}\right) \nu=\tau\left(B^{-1}\right) \nu .
$$

Since $\tau\left(B^{-1}\right)=[\rho(B)]^{-1}>0$, from the above formula we have $\left(B^{-1}\right)^{T} v$ is a positive vector, then $C=V B^{-1}=\left(w_{i j} v_{i}\right)$ is a strictly diagonally dominant matrix by columns, where $V=$ $\operatorname{diag}\left(v_{1}, v_{2}, \ldots, v_{n}\right)$. Notice that $C^{-1}=B V^{-1}=\left(\frac{b_{i j}}{v_{j}}\right)_{n \times n}$, then by Lemma 2.2(ii), for $i \neq j$, we have

$$
\frac{b_{i j}}{v_{j}} \leq \frac{\sum_{k=1, k \neq j}^{n}\left|w_{k j}\right| v_{k}}{w_{j j} v_{j}} \frac{b_{i i}}{v_{i}}=\frac{-\sum_{k=1, k \neq j}^{n} w_{k j} v_{k}}{w_{j j} v_{j}} \frac{b_{i i}}{v_{i}}=\frac{\left(w_{j j}-\tau\left(B^{-1}\right)\right) v_{j}}{w_{j j} v_{j}} \frac{b_{i i}}{v_{i}}
$$

Thus

$$
b_{i j} \leq \frac{\left(w_{j j}-\tau\left(B^{-1}\right)\right) v_{j} b_{i i}}{w_{j j} v_{i}}=\frac{b_{i i}}{v_{i}} g_{j} u_{j},
$$

where $g_{j}=\frac{\left(w_{j j}-\tau\left(B^{-1}\right)\right) v_{j}}{w_{j j} u_{j}}$. Let $G=\operatorname{diag}\left(g_{1}, g_{2}, \ldots, g_{n}\right)$, by Lemma 2.3 we have $G$ is a positive diagonal matrix and

$$
G(A \circ B) G^{-1}=\left(\begin{array}{cccc}
a_{11} b_{11} & \frac{g_{1} a_{12} b_{12}}{g_{2}} & \ldots & \frac{g_{1} a_{11} b_{1 n}}{g_{n}} \\
\frac{g_{2} a_{21} b_{21}}{g_{1}} & a_{22} b_{22} & \ldots & \frac{g_{2} a_{22} b_{2 n}}{g_{n}} \\
\vdots & \vdots & \ddots & \vdots \\
\frac{g_{n} a_{n 1} b_{n 1}}{g_{1}} & \frac{g_{n} a_{n 2} b_{n 2}}{g_{2}} & \ldots & a_{n n} b_{n n}
\end{array}\right) .
$$

Hence, $\rho(A \circ B)=\rho\left(G(A \circ B) G^{-1}\right)$, then by Lemma 2.4 we have

$$
\rho(A \circ B) \leq \max _{i \neq j} \frac{1}{2}\left\{a_{i i} b_{i i}+a_{j j} b_{j j}+\left[\left(a_{i i} b_{i i}-a_{j j} b_{j j}\right)^{2}+4 \sum_{k=1, k \neq i}^{n} \frac{g_{i} a_{i k} b_{i k}}{g_{k}} \sum_{k=1, k \neq j}^{n} \frac{g_{j} a_{j k} b_{j k}}{g_{k}}\right]^{\frac{1}{2}}\right\}
$$

and

$$
\begin{aligned}
& \sum_{k=1, k \neq i}^{n} \frac{g_{i} a_{i k} b_{i k}}{g_{k}} \sum_{k=1, k \neq j}^{n} \frac{g_{j} a_{j k} b_{j k}}{g_{k}} \\
& \leq\left(g_{i} \frac{b_{i i}}{v_{i}} \sum_{k=1, k \neq i}^{n} a_{i k} u_{k}\right)\left(g_{j} \frac{b_{j j}}{v_{j}} \sum_{k=1, k \neq j}^{n} a_{j k} u_{k}\right) \\
& \quad=g_{i} \frac{b_{i i}}{v_{i}}\left(\rho(A)-a_{i i}\right) u_{i} g_{j} \frac{b_{j j}}{v_{j}}\left(\rho(A)-a_{j j}\right) u_{j}
\end{aligned}
$$




$$
\begin{aligned}
& =\frac{\left(w_{i i}-\tau\left(B^{-1}\right)\right) v_{i}}{w_{i i} u_{i}} \frac{b_{i i}}{v_{i}}\left(\rho(A)-a_{i i}\right) u_{i} \frac{\left(w_{i j}-\tau\left(B^{-1}\right)\right) v_{j}}{w_{j j} u_{j}} \frac{b_{i j}}{v_{j}}\left(\rho(A)-a_{j j}\right) u_{j} \\
& =\frac{b_{i i} b_{i j}}{w_{i i} w_{j j}}\left(\rho(A)-a_{i i}\right)\left(\rho(A)-a_{j j}\right)\left(w_{i i}-\tau\left(B^{-1}\right)\right)\left(w_{j j}-\tau\left(B^{-1}\right)\right) \\
& =\frac{b_{i i} b_{i j}}{w_{i i} w_{j j}}\left(\rho(A)-a_{i i}\right)\left(\rho(A)-a_{j j}\right)\left(w_{i i}-\frac{1}{\rho(B)}\right)\left(w_{j j}-\frac{1}{\rho(B)}\right) .
\end{aligned}
$$

Thus, from the above two inequalities, we have

$$
\begin{aligned}
\rho(A \circ B) \leq & \max _{i \neq j} \frac{1}{2}\left\{a_{i i} b_{i i}+a_{j j} b_{j j}+\left[\left(a_{i i} b_{i i}-a_{j j} b_{i j}\right)^{2}\right.\right. \\
& \left.\left.+4 \frac{b_{i i} b_{i j}}{w_{i i} w_{j j}}\left[\rho(A)-a_{i i}\right]\left[\rho(A)-a_{j j}\right]\left[w_{i i}-\frac{1}{\rho(B)}\right]\left[w_{j j}-\frac{1}{\rho(B)}\right]\right]^{\frac{1}{2}}\right\} .
\end{aligned}
$$

Case 2: If $A \circ B$ is reducible, then more than one of $A$ and $B$ is reducible. By Lemma 2.6 we know that all leading principal minors of $B^{-1}$ are positive. If we denote by $P=\left(p_{i j}\right)$ the $n \times n$ permutation matrix with $p_{12}=p_{23}=\cdots=p_{n-1, n}=p_{n, 1}=1$, the remaining $p_{i j}$ being zero, then, for any chosen positive real number $\varepsilon$, sufficiently small such that all the leading principal minors of $\left(B^{-1}-\varepsilon P\right)^{-1}$ are positive, then $A+\varepsilon P$ and $\left(B^{-1}-\varepsilon P\right)^{-1}$ are irreducible nonnegative matrix and irreducible inverse $M$-matrix, respectively. Now we substitute $A+\varepsilon P$ and $\left(B^{-1}-\varepsilon P\right)^{-1}$ for $A$ and $B$, respectively, in the previous case, and then letting $\varepsilon \rightarrow 0$, the result follows by continuity.

Remark 3.1 The upper bound in Theorem 3.1 is an improvement on Theorem 2 in [6]. We next give a simple comparison between the upper bound in Theorem 3.1 and the bound derived in (1). Without loss of generality, for $i \neq j$, assume that

$$
a_{i i} b_{i i}+\frac{b_{i i}}{w_{i i}}\left[\rho(A)-a_{i i}\right]\left[w_{i i}-\frac{1}{\rho(B)}\right] \geq a_{i j} b_{j j}+\frac{b_{j j}}{w_{j j}}\left[\rho(A)-a_{j j}\right]\left[w_{j j}-\frac{1}{\rho(B)}\right] .
$$

Thus, we can write the above equivalently as

$$
a_{i i} b_{i i}-a_{j j} b_{j j}+\frac{b_{i i}}{w_{i i}}\left[\rho(A)-a_{i i}\right]\left[w_{i i}-\tau\left(B^{-1}\right)\right] \geq \frac{b_{i j}}{w_{j j}}\left[\rho(A)-a_{j j}\right]\left[w_{j j}-\tau\left(B^{-1}\right)\right] .
$$

Thus, we have

$$
\begin{gathered}
\left(a_{i i} b_{i i}-a_{j j} b_{j j}\right)^{2}+4 \frac{b_{i i} b_{j j}}{w_{i i} w_{j j}}\left[\rho(A)-a_{i i}\right]\left[\rho(A)-a_{j j}\right]\left[w_{i i}-\frac{1}{\rho(B)}\right]\left[w_{i j}-\frac{1}{\rho(B)}\right] \\
\leq\left(a_{i i} b_{i i}-a_{i j} b_{j j}\right)^{2}+4 \frac{b_{i i}}{w_{i i}}\left[\rho(A)-a_{i i}\right]\left[w_{i i}-\tau\left(B^{-1}\right)\right]\left(a_{i i} b_{i i}-a_{j j} b_{j j}\right) \\
\quad+4\left[\frac{b_{i i}}{w_{i i}}\left[\rho(A)-a_{i i}\right]\left[w_{i i}-\tau\left(B^{-1}\right)\right]\right]^{2} \\
=\left(a_{i i} b_{i i}-a_{i j} b_{j j}+2 \frac{b_{i i}}{w_{i i}}\left[\rho(A)-a_{i i}\right]\left[w_{i i}-\tau\left(B^{-1}\right)\right]\right)^{2} .
\end{gathered}
$$


Then we have

$$
\begin{aligned}
\rho(A \circ B) \leq & \max _{i \neq j} \frac{1}{2}\left\{a_{i i} b_{i i}+a_{i j} b_{j j}+\left[\left(a_{i i} b_{i i}-a_{i j} b_{j j}\right)^{2}\right.\right. \\
& \left.\left.+4 \frac{b_{i i} b_{j j}}{w_{i i} w_{j j}}\left[\rho(A)-a_{i i}\right]\left[\rho(A)-a_{i j}\right]\left[w_{i i}-\frac{1}{\rho(B)}\right]\left[w_{j j}-\frac{1}{\rho(B)}\right]\right]^{\frac{1}{2}}\right\} \\
\leq & \max _{i \neq j} \frac{1}{2}\left\{a_{i i} b_{i i}+a_{i j} b_{j j}+a_{i i} b_{i i}-a_{j j} b_{j j}\right. \\
& \left.+2 \frac{b_{i i}}{w_{i i}}\left[\rho(A)-a_{i i}\right]\left[w_{i i}-\tau\left(B^{-1}\right)\right]\right\} \\
= & \max _{1 \leq i \leq n} \frac{1}{2}\left\{2 a_{i i} b_{i i}+2 \frac{b_{i i}}{w_{i i}}\left[\rho(A)-a_{i i}\right]\left[w_{i i}-\tau\left(B^{-1}\right)\right]\right\} \\
= & \max _{1 \leq i \leq n}\left\{a_{i i} b_{i i}+\frac{b_{i i}}{w_{i i}}\left[\rho(A)-a_{i i}\right]\left[w_{i i}-\tau\left(B^{-1}\right)\right]\right\} \\
= & \rho(A) \tau\left(B^{-1}\right) \max _{1 \leq i \leq n}\left[\left(\frac{a_{i i}}{\rho(A)}+\frac{w_{i i}}{\tau\left(B^{-1}\right)}-1\right) \frac{b_{i i}}{w_{i i}}\right] \\
= & \frac{\rho(A)}{\rho(B)} \max _{1 \leq i \leq n}\left[\left(\frac{a_{i i}}{\rho(A)}+w_{i i} \rho(B)-1\right) \frac{b_{i i}}{w_{i i}}\right] .
\end{aligned}
$$

This shows that Theorem 3.1 in this paper is an improvement on Theorem 2 in [6].

\section{Example 3.1 Let}

$$
A=\left(\begin{array}{lll}
1 & 1 & 1 \\
1 & 2 & 1 \\
1 & 1 & 3
\end{array}\right), \quad B=\left(\begin{array}{ccc}
1.2 & 0.4 & 0.4 \\
0.4 & 1.2 & 0.4 \\
0.4 & 0.4 & 1.2
\end{array}\right)
$$

where $A$ is a nonnegative matrix and $B$ is an inverse $M$-matrix. By Theorem 2 in [6], we have

$$
\rho(A \circ B) \leq 4.3285 .
$$

By Theorem 3.1 in this paper, we get

$$
\rho(A \circ B) \leq 4.1524 .
$$

The example shows that the bound in Theorem 3.1 is better than the existing bound in Theorem 2 in [6]. In fact, $\rho(A \circ B)=3.8178$.

Theorem 3.2 Let $A=\left(a_{i j}\right) \in \mathbb{R}^{n \times n}$ and $B=\left(b_{i j}\right) \in \mathbb{R}^{n \times n}$ be nonnegative matrices, then

$$
\begin{aligned}
\rho(A \circ B) \leq & \max _{i \neq j} \frac{1}{2}\left\{a_{i i} b_{i i}+a_{j j} b_{j j}+\left[\left(a_{i i} b_{i i}-a_{j j} b_{j j}\right)^{2}\right.\right. \\
& \left.\left.+4\left[\alpha_{i} \alpha_{j} \beta_{i} \beta_{j}\left(\rho(A)-a_{i i}\right)\left(\rho(A)-a_{j j}\right)\left(\rho(B)-b_{i i}\right)\left(\rho(B)-b_{j j}\right)\right]^{\frac{1}{2}}\right]^{\frac{1}{2}}\right\},
\end{aligned}
$$

where $\alpha_{i}=\max _{k \neq i}\left\{\left|a_{i k}\right|\right\}, \beta_{i}=\max _{k \neq i}\left\{\left|b_{i k}\right|\right\}(i \in N)$. 
Proof It is easy to see that conclusion in Theorem 3.2 holds with equality for $n=1$. Next, we assume that $n \geq 2$. Two cases will be discussed in the following.

Case 1: If $A \circ B$ is irreducible, then $A$ and $B$ are irreducible. Since $A \geq O$ and $B \geq O$, then there exist two positive vectors $t=\left(t_{1}^{2}, t_{2}^{2}, \ldots, t_{n}^{2}\right)^{T}$ and $f=\left(f_{1}^{2}, f_{2}^{2}, \ldots, f_{n}^{2}\right)^{T}$ such that

$$
A t=\rho(A) t, \quad B f=\rho(B) f .
$$

Hence, we have

$$
\sum_{j=1, j \neq i}^{n} a_{i j} t_{j}^{2}=\left[\rho(A)-a_{i i}\right] t_{i}^{2}, \quad \sum_{j=1, j \neq i}^{n} b_{i j} f_{j}^{2}=\left[\rho(B)-b_{i i}\right] f_{i}^{2} .
$$

Let $\alpha_{i}=\max _{k \neq i}\left\{\left|a_{i k}\right|\right\}$ and $\beta_{i}=\max _{k \neq i}\left\{\left|b_{i k}\right|\right\}$ for all $i \in N$. Define a positive diagonal matrix $W=\operatorname{diag}\left(t_{1} f_{1}, t_{2} f_{2}, \ldots, t_{n} f_{n}\right)$, then

$$
W^{-1}(A \circ B) W=\left(\begin{array}{cccc}
a_{11} b_{11} & \frac{a_{12} b_{12} t_{2} f_{2}}{t_{1} f_{1}} & \ldots & \frac{a_{1 n} b_{1 n} t_{n} f_{n}}{t_{1} f_{1}} \\
\frac{a_{21} b_{21} t_{1} f_{1}}{t_{2} f_{2}} & a_{22} b_{22} & \ldots & \frac{a_{2 n} b_{2 n} n_{n} f_{n}}{t_{2} f_{2}} \\
\vdots & \vdots & \ddots & \vdots \\
\frac{a_{n 1} b_{n 1} t_{1} f_{1}}{t_{n} f_{n}} & \frac{a_{n 2} b_{n 2} t_{2} f_{2}}{t_{n} f_{n}} & \ldots & a_{n n} b_{n n}
\end{array}\right) .
$$

Since $\rho(A \circ B)=\rho\left(W^{-1}(A \circ B) W\right)$, let $C=W^{-1}(A \circ B) W$, then $C$ is a nonnegative matrix, by Lemma 2.4 we have

$$
\begin{aligned}
\rho(A \circ B) \leq & \max _{i \neq j} \frac{1}{2}\left\{a_{i i} b_{i i}+a_{j j} b_{j j}\right. \\
& \left.+\left[\left(a_{i i} b_{i i}-a_{j j} b_{j j}\right)^{2}+4 \sum_{k=1, k \neq i}^{n} \frac{a_{i k} b_{i k} t_{k} f_{k}}{t_{i} f_{i}} \sum_{k=1, k \neq j}^{n} \frac{a_{j k} b_{j k} t_{k} f_{k}}{t_{j} f_{j}}\right]^{\frac{1}{2}}\right\}
\end{aligned}
$$

and

$$
\begin{aligned}
& \sum_{k=1, k \neq i}^{n} \frac{a_{i k} b_{i k} t_{k} f_{k}}{t_{i} f_{i}} \sum_{k=1, k \neq j}^{n} \frac{a_{j k} b_{j k} t_{k} f_{k}}{t_{j} f_{j}} \\
& \quad \leq\left(\sum_{k=1, k \neq i}^{n} \frac{a_{i k}^{2} t_{k}^{2}}{t_{i}^{2}}\right)^{\frac{1}{2}}\left(\sum_{k=1, k \neq i}^{n} \frac{b_{i k}^{2} f_{k}^{2}}{f_{i}^{2}}\right)^{\frac{1}{2}}\left(\sum_{k=1, k \neq j}^{n} \frac{a_{j k}^{2} t_{k}^{2}}{t_{j}^{2}}\right)^{\frac{1}{2}}\left(\sum_{k=1, k \neq j}^{n} \frac{b_{j k}^{2} f_{k}^{2}}{f_{j}^{2}}\right)^{\frac{1}{2}} \\
& \quad \leq\left[\left(\alpha_{i} \alpha_{j} \beta_{i} \beta_{j}\right)\left(\sum_{k=1, k \neq i}^{n} \frac{a_{i k} t_{k}^{2}}{t_{i}^{2}}\right)\left(\sum_{k=1, k \neq i}^{n} \frac{b_{i k} f_{k}^{2}}{f_{i}^{2}}\right)\left(\sum_{k=1, k \neq j}^{n} \frac{a_{j k} t_{k}^{2}}{t_{j}^{2}}\right)\left(\sum_{k=1, k \neq j}^{n} \frac{b_{j k} f_{k}^{2}}{f_{j}^{2}}\right)\right]^{\frac{1}{2}} \\
& \quad=\left[\left(\alpha_{i} \alpha_{j} \beta_{i} \beta_{j}\right)\left(\rho(A)-a_{i i}\right)\left(\rho(B)-b_{i i}\right)\left(\rho(A)-a_{j j}\right)\left(\rho(B)-b_{j j}\right)\right]^{\frac{1}{2}} .
\end{aligned}
$$

Thus, from the above two inequalities, we have

$$
\begin{aligned}
\rho(A \circ B) \leq & \max _{i \neq j} \frac{1}{2}\left\{a_{i i} b_{i i}+a_{i j} b_{j j}+\left[\left(a_{i i} b_{i i}-a_{i j} b_{j j}\right)^{2}\right.\right. \\
& \left.\left.+4\left[\alpha_{i} \alpha_{j} \beta_{i} \beta_{j}\left(\rho(A)-a_{i i}\right)\left(\rho(A)-a_{j j}\right)\left(\rho(B)-b_{i i}\right)\left(\rho(B)-b_{j j}\right)\right]^{\frac{1}{2}}\right]^{\frac{1}{2}}\right\} .
\end{aligned}
$$


Case 2: If $A \circ B$ is reducible, then more than one of $A$ and $B$ is reducible. If we denote by $P=\left(p_{i j}\right)$ the $n \times n$ permutation matrix with $p_{12}=p_{23}=\cdots=p_{n-1, n}=p_{n, 1}=1$, the remaining $p_{i j}$ being zero, then, for any chosen positive real number $\varepsilon$, sufficiently small such that $A+\varepsilon P$ and $B+\varepsilon P$ are irreducible nonnegative matrices. Now we substitute $A+\varepsilon P$ and $B+\varepsilon P$ for $A$ and $B$, respectively, in the previous case, and then letting $\varepsilon \rightarrow 0$, the result follows by continuity.

Example 3.2 [10] Let

$$
A=\left(\begin{array}{cccc}
4 & 1 & 0 & 2 \\
1 & 0.05 & 1 & 1 \\
0 & 1 & 4 & 0.5 \\
1 & 0.5 & 0 & 4
\end{array}\right), \quad B=\left(\begin{array}{cccc}
1 & 1 & 1 & 1 \\
1 & 1 & 1 & 1 \\
1 & 1 & 1 & 1 \\
1 & 1 & 1 & 1
\end{array}\right)
$$

It is easy to verify that $A$ and $B$ are nonnegative matrices. By Theorem 4 in [11], we have

$$
\rho(A \circ B) \leq 17.1017
$$

By Theorem 4 in [10], we have

$$
\rho(A \circ B) \leq 11.6478 .
$$

By Theorem 3.2 in this paper, we get

$$
\rho(A \circ B) \leq 6.7123
$$

The example shows that the bound in Theorem 3.2 is better than the existing bounds in Theorem 4 in [11] and Theorem 4 in [10] in some cases. In fact, $\rho(A \circ B)=5.7339$.

In 2013, Guo et al. [9] obtained the following result:

Let $A=\left(a_{i j}\right) \in \mathbb{R}^{n \times n}, B=\left(b_{i j}\right) \in \mathbb{R}^{n \times n}$ be nonnegative matrices, then

$$
\rho(A \circ B) \leq \max _{1 \leq i \leq n}\left\{a_{i i} b_{i i}+s_{i} \sum_{j=1, j \neq i}^{n} \frac{b_{j i}}{m_{j}}\right\},
$$

where

$$
\begin{aligned}
& r_{l i}=\frac{\left|a_{l i}\right|}{\left|a_{l l}\right|-\sum_{k=1, k \neq l, i}^{n}\left|a_{l k}\right|} \quad(l \neq i), \quad r_{i}=\max _{l \neq i}\left\{r_{l i}\right\} \quad(i \in N), \\
& s_{j i}=\left|a_{j i}\right| m_{j}, \quad m_{j}=\left\{\begin{array}{ll}
r_{j} & \text { if } r_{j} \neq 0, \\
1 & \text { if } r_{j}=0,
\end{array} s_{j}=\max _{j \neq i}\left\{s_{j i}\right\} \quad(i, j \in N) .\right.
\end{aligned}
$$

But the result in the above formula does not apply for all spectral radius of the Hadamard product of nonnegative matrices. There is a counterexample as follows. 


\section{Example 3.3 Let}

$$
A=\left(\begin{array}{lll}
6 & 4 & 4 \\
4 & 2 & 4 \\
4 & 4 & 2
\end{array}\right), \quad B=\left(\begin{array}{lll}
1 & 1 & 1 \\
1 & 1 & 1 \\
1 & 1 & 1
\end{array}\right)
$$

It is easy to verify that $A$ and $B$ are nonnegative matrices. By Theorem 2.1 in [9], we have

$$
\rho(A \circ B) \leq \max _{1 \leq i \leq n}\left\{a_{i i} b_{i i}+s_{i} \sum_{j=1, j \neq i}^{n} \frac{b_{j i}}{m_{j}}\right\}=2 .
$$

But in fact, $\rho(A \circ B)=11.6569$. So inequality in (2) does not hold in this example. Thus the upper bound in (2) does not apply for all spectral radius of the Hadamard product of nonnegative matrices. Since the proof in Theorem 2.1 in [9], i.e., mainly applied Lemma 2.7, and $r_{l i}<0(l \neq i)$ when $\left|a_{l l}\right|-\sum_{k=1, k \neq l, i}^{n}\left|a_{l k}\right|<0$, which does not confirm that conditions $x_{1}, x_{2}, \ldots, x_{n}$ are positive in Lemma 2.7 .

Next, we present a new upper bound on $\rho(A \circ B)$, which improves the result in (2).

Theorem 3.3 Let $A=\left(a_{i j}\right) \in \mathbb{R}^{n \times n}$ and $B=\left(b_{i j}\right) \in \mathbb{R}^{n \times n}$ be nonnegative matrices, related expressions the same as to (2), if $r_{i} \geq 0$ for all $i \in N$, then

$$
\rho(A \circ B) \leq \max _{\gamma \in \zeta(A \circ B)}\left[\prod_{i \in \bar{\gamma}}\left(a_{i i} b_{i i}+s_{i} \sum_{j=1, j \neq i}^{n} \frac{b_{j i}}{m_{j}}\right)\right]^{\frac{1}{|\gamma|}} .
$$

Proof Case 1: If $A \circ B$ is irreducible, then $A$ and $B$ are irreducible, since $\rho(A \circ B)=\rho((A \circ$ $\left.B)^{T}\right)$. Let $S=\operatorname{diag}\left(s_{1}, s_{2}, \ldots, s_{n}\right)$, then $S$ is a positive diagonal matrix, then

$$
\begin{aligned}
R_{i}^{\prime}\left(S^{-1}(A \circ B)^{T} S\right) & =a_{i i} b_{i i}+s_{i} \sum_{j=1, j \neq i}^{n} \frac{a_{j i} b_{j i}}{s_{j}} \\
& \leq a_{i i} b_{i i}+s_{i} \sum_{j=1, j \neq i}^{n} \frac{a_{j i} b_{j i}}{\left|a_{j i}\right| m_{j}} \\
& =a_{i i} b_{i i}+s_{i} \sum_{j=1, j \neq i}^{n} \frac{b_{j i}}{m_{j}} .
\end{aligned}
$$

By Lemma 2.5, we have

$$
\rho(A \circ B)=\rho\left((A \circ B)^{T}\right) \leq \max _{\gamma \in \zeta(A \circ B)}\left[\prod_{i \in \bar{\gamma}}\left(a_{i i} b_{i i}+s_{i} \sum_{j=1, j \neq i}^{n} \frac{b_{j i}}{m_{j}}\right)\right]^{\frac{1}{|\gamma|}} .
$$

Case 2: If $A \circ B$ is reducible, then more than one of $A$ and $B$ is reducible. If we denote by $P=\left(p_{i j}\right)$ the $n \times n$ permutation matrix with $p_{12}=p_{23}=\cdots=p_{n-1, n}=p_{n, 1}=1$, the remaining $p_{i j}$ being zero, then, for any chosen positive real number $\varepsilon$, sufficiently small such that $A+\varepsilon P$ and $B+\varepsilon P$ are irreducible nonnegative matrices. Now we substitute $A+\varepsilon P$ and 
$B+\varepsilon P$ for $A$ and $B$, respectively, in the previous case, and then letting $\varepsilon \rightarrow 0$, the result follows by continuity.

Remark 3.2 The upper bound in Theorem 3.3 is an improvement on Theorem 2.1 in [9]. We next give a simple comparison between the upper bound in Theorem 3.3 and the bound in (2). In fact, it is easy to see

$$
\begin{aligned}
\rho(A \circ B) & \leq \max _{\gamma \in \zeta(A \circ B)}\left[\prod_{i \in \bar{\gamma}}\left(a_{i i} b_{i i}+s_{i} \sum_{j=1, j \neq i}^{n} \frac{b_{j i}}{m_{j}}\right)\right]^{\frac{1}{|\gamma|}} \\
& \leq \max _{\gamma \in \zeta(A \circ B)}\left[\prod_{i \in \bar{\gamma}} \max _{1 \leq i \leq n}\left(a_{i i} b_{i i}+s_{i} \sum_{j=1, j \neq i}^{n} \frac{b_{j i}}{m_{j}}\right)\right]^{\frac{1}{|\gamma|}} \\
& =\max _{1 \leq i \leq n}\left(a_{i i} b_{i i}+s_{i} \sum_{j=1, j \leq i}^{n} \frac{b_{j i}}{m_{j}}\right) .
\end{aligned}
$$

This shows that Theorem 3.3 is an improvement on Theorem 2.1 in [9].

Example 3.4 [9] Let

$$
A=\left(\begin{array}{llll}
4 & 1 & 1 & 1 \\
2 & 5 & 1 & 1 \\
0 & 2 & 4 & 1 \\
1 & 1 & 1 & 4
\end{array}\right), \quad B=\left(\begin{array}{llll}
1 & 1 & 0 & 0 \\
1 & 3 & 2 & 0 \\
0 & 1 & 4 & 3 \\
0 & 0 & 1 & 5
\end{array}\right) .
$$

It is easy to verify that $A$ and $B$ are nonnegative matrices and $r_{i}>0$ for all $i \in N$. By Theorem 4 in [9], we have

$$
\rho(A \circ B) \leq 25.5364 \text {. }
$$

By Theorem 4 in [10], we have

$$
\rho(A \circ B) \leq 25.3644 .
$$

By Theorem 2.1 in [9], we have

$$
\rho(A \circ B) \leq 24
$$

By Theorem 2.2 in [9], we have

$$
\rho(A \circ B) \leq 22.1633
$$

By Theorem 3.3 in this paper, we get

$$
\rho(A \circ B) \leq 21.9773 \text {. }
$$

The example shows that the bound in Theorem 3.3 is better than the existing bounds in Theorem 4 in [11], Theorem 4 in [10] and Theorems 2.1, 2.2 in [9]. In fact, $\rho(A \circ B) \leq$ 20.7439 . 


\section{Upper and lower bounds for the spectral radius of $J_{A}$}

In 2008, Huang [2] obtained the following result:

Let $A=\left(a_{i j}\right) \in \mathbb{R}^{n \times n}$ be a nonsingular $M$-matrix, then

$$
\min _{1 \leq i \leq n}\left(1-\frac{\tau(A)}{a_{i i}}\right) \leq \rho\left(J_{A}\right) \leq \max _{1 \leq i \leq n}\left(1-\frac{\tau(A)}{a_{i i}}\right) .
$$

In 2010, Liu et al. [3] improved the bounds in (3) and obtained the following result:

Let $A=\left(a_{i j}\right) \in \mathbb{R}^{n \times n}$ be an irreducible nonsingular $M$-matrix, then

$$
\min _{i \neq j} \sqrt{\left(1-\frac{\tau(A)}{a_{i i}}\right)\left(1-\frac{\tau(A)}{a_{j j}}\right)} \leq \rho\left(J_{A}\right) \leq \max _{i \neq j} \sqrt{\left(1-\frac{\tau(A)}{a_{i i}}\right)\left(1-\frac{\tau(A)}{a_{j j}}\right)} .
$$

Next, we present some new upper and lower bounds for $\rho\left(J_{A}\right)$, the bounds improved the results in (3) and (4).

Theorem 4.1 Let $A=\left(a_{i j}\right) \in \mathbb{R}^{n \times n}$ be a nonsingular M-matrix, then

$$
\min _{\left.\gamma \in \zeta J_{A}\right)}\left[\prod_{i \in \bar{\gamma}}\left(1-\frac{\tau(A)}{a_{i i}}\right)\right]^{\frac{1}{|\gamma|}} \leq \rho\left(J_{A}\right) \leq \max _{\left.\gamma \in \zeta J_{A}\right)}\left[\prod_{i \in \bar{\gamma}}\left(1-\frac{\tau(A)}{a_{i i}}\right)\right]^{\frac{1}{|\gamma|}} .
$$

Proof Case 1: If $A$ is an irreducible nonsingular $M$-matrix, then $A^{-1}>0$, then there exists a positive vector $u=\left(u_{1}, u_{2}, \ldots, u_{n}\right)^{T}$ such that $A u=\tau(A) u$. Thus, for all $i \in N$, we have

$$
\tau(A)=\frac{\sum_{j=1}^{n} a_{i j} u_{j}}{u_{i}}
$$

i.e.,

$$
a_{i i}-\tau(A)=\frac{\sum_{j=1, j \neq i}^{n}\left|a_{i j}\right| u_{j}}{u_{i}}
$$

Let $U=\operatorname{diag}\left(u_{1}, u_{2}, \ldots, u_{n}\right)$, then $U$ is a positive diagonal matrix, then

$$
R_{i}^{\prime}\left(U^{-1} J_{A} U\right)=\frac{\sum_{j=1, j \neq i}^{n}\left|a_{i j}\right| u_{j}}{a_{i i} u_{i}}=1-\frac{\tau(A)}{a_{i i}} .
$$

By Lemma 2.5, we have

$$
\min _{\left.\gamma \in \zeta U_{A}\right)}\left[\prod_{i \in \bar{\gamma}}\left(1-\frac{\tau(A)}{a_{i i}}\right)\right]^{\frac{1}{|\gamma|}} \leq \rho\left(J_{A}\right) \leq \max _{\left.\gamma \in \zeta D_{A}\right)}\left[\prod_{i \in \bar{\gamma}}\left(1-\frac{\tau(A)}{a_{i i}}\right)\right]^{\frac{1}{|\gamma|}} .
$$

Case 2: If $A$ is reducible, by Lemma 2.6 we know that all leading principal minors of $A$ are positive. If we denote by $P=\left(p_{i j}\right)$ the $n \times n$ permutation matrix with $p_{12}=p_{23}=\cdots=$ $p_{n-1, n}=p_{n, 1}=1$, the remaining $p_{i j}$ being zero, then, for any chosen positive real number $\varepsilon$, sufficiently small such that all the leading principal minors of $A-\varepsilon P$ are positive, $A-\varepsilon P$ is an irreducible nonsingular $M$-matrix. Now we substitute $A-\varepsilon P$ for $A$ in the previous case, and then letting $\varepsilon \rightarrow 0$, the result follows by continuity. 
Remark 4.1 The bounds in Theorem 4.1 are sharper than those in Theorem 1 in [2]. In fact, by Lemma 2.8 , we have $1-\frac{\tau(A)}{a_{i i}} \geq 0$ for all $i \in N$, then

$$
\begin{aligned}
\rho\left(J_{A}\right) & \leq \max _{\left.\gamma \in \zeta J_{A}\right)}\left[\prod_{i \in \bar{\gamma}}\left(1-\frac{\tau(A)}{a_{i i}}\right)\right]^{\frac{1}{|\gamma|}} \\
& \leq \max _{\left.\gamma \in \zeta V_{A}\right)}\left[\prod_{i \in \bar{\gamma}} \max _{1 \leq i \leq n}\left(1-\frac{\tau(A)}{a_{i i}}\right)\right]^{\frac{1}{|\gamma|}}=\max _{1 \leq i \leq n}\left(1-\frac{\tau(A)}{a_{i i}}\right)
\end{aligned}
$$

and

$$
\begin{aligned}
\rho\left(J_{A}\right) & \geq \min _{\left.\gamma \in \zeta U_{A}\right)}\left[\prod_{i \in \bar{\gamma}}\left(1-\frac{\tau(A)}{a_{i i}}\right)\right]^{\frac{1}{|\gamma|}} \\
& \geq \min _{\left.\gamma \in \zeta U_{A}\right)}\left[\prod_{i \in \bar{\gamma}} \min _{1 \leq i \leq n}\left(1-\frac{\tau(A)}{a_{i i}}\right)\right]^{\frac{1}{|\gamma|}}=\min _{1 \leq i \leq n}\left(1-\frac{\tau(A)}{a_{i i}}\right) .
\end{aligned}
$$

This shows that Theorem 4.1 is an improvement on Theorem 1 in [2]. The bounds in Theorem 4.1 are sharper than the bounds derived in Theorem 1 in [3]. Without loss of generality, assume that two circuits $\gamma^{\prime}: h_{1} \rightarrow h_{2} \rightarrow \cdots \rightarrow h_{k} \rightarrow h_{1}(k \geq 2)$ and $\xi^{\prime}: z_{1} \rightarrow$ $z_{2} \rightarrow \cdots \rightarrow z_{t} \rightarrow z_{1}(t \geq 2)$ in $J_{A}$ such that

$$
\left[\prod_{i=h_{1}}^{h_{k}}\left(1-\frac{\tau(A)}{a_{i i}}\right)\right]^{\frac{1}{k}}=\max _{\left.\gamma \in \zeta U_{A}\right)}\left[\prod_{i \in \bar{\gamma}}\left(1-\frac{\tau(A)}{a_{i i}}\right)\right]^{\frac{1}{|\gamma|}}
$$

and

$$
\left[\prod_{i=z_{1}}^{z_{t}}\left(1-\frac{\tau(A)}{a_{i i}}\right)\right]^{\frac{1}{t}}=\min _{\gamma \in \zeta\left(V_{A}\right)}\left[\prod_{i \in \bar{\gamma}}\left(1-\frac{\tau(A)}{a_{i i}}\right)\right]^{\frac{1}{|\gamma|}}
$$

respectively. Since

$$
\begin{aligned}
{\left[\prod_{i=h_{1}}^{h_{k}}\left(1-\frac{\tau(A)}{a_{i i}}\right)^{2}\right]^{\frac{1}{k}}=} & {\left[\left(1-\frac{\tau(A)}{a_{h_{1} h_{1}}}\right)\left(1-\frac{\tau(A)}{a_{h_{2} h_{2}}}\right) \cdots\left(1-\frac{\tau(A)}{a_{h_{k-1} h_{k-1}}}\right)\right.} \\
& \left.\times\left(1-\frac{\tau(A)}{a_{h_{k} h_{k}}}\right)\left(1-\frac{\tau(A)}{a_{h_{k} h_{k}}}\right)\left(1-\frac{\tau(A)}{a_{h_{1} h_{1}}}\right)\right]^{\frac{1}{k}} \\
\leq & \left\{\left[\max _{i \neq j}\left(1-\frac{\tau(A)}{a_{i i}}\right)\left(1-\frac{\tau(A)}{a_{i j}}\right)\right]^{k}\right\}^{\frac{1}{k}} \\
= & \max _{i \neq j}\left(1-\frac{\tau(A)}{a_{i i}}\right)\left(1-\frac{\tau(A)}{a_{j j}}\right),
\end{aligned}
$$

then

$$
\max _{\left.\gamma \in \zeta D_{A}\right)}\left[\prod_{i \in \bar{\gamma}}\left(1-\frac{\tau(A)}{a_{i i}}\right)\right]^{\frac{1}{|\gamma|}} \leq \max _{i \neq j} \sqrt{\left(1-\frac{\tau(A)}{a_{i i}}\right)\left(1-\frac{\tau(A)}{a_{j j}}\right)} .
$$


Similarly, we can prove that

$$
\min _{\left.\gamma \in \zeta U_{A}\right)}\left[\prod_{i \in \bar{\gamma}}\left(1-\frac{\tau(A)}{a_{i i}}\right)\right]^{\frac{1}{|\gamma|}} \geq \min _{i \neq j} \sqrt{\left(1-\frac{\tau(A)}{a_{i i}}\right)\left(1-\frac{\tau(A)}{a_{j j}}\right)} .
$$

This shows that Theorem 4.1 is an improvement on Theorem 1 in [3].

\section{Example 4.1 Let}

$$
A=\left(\begin{array}{ccc}
2 & -1 & -1 \\
-2 & 3 & 0 \\
-1 & 0 & 4
\end{array}\right)
$$

It is easy to verify that $A$ is a nonsingular $M$-matrix. By Theorem 1 in [2], we have

$$
0.6072 \leq \rho\left(J_{A}\right) \leq 0.8036
$$

By Theorem 1 in [3], we have

$$
0.6694 \leq \rho\left(J_{A}\right) \leq 0.7701 \text {. }
$$

By Theorem 4.1 in this paper, we get

$$
0.6694 \leq \rho\left(J_{A}\right) \leq 0.6985 \text {. }
$$

The example shows that the bounds in Theorem 4.1 are better than the existing bounds in Theorem 1 in [2] and Theorem 1 in [3]. In fact, $\rho\left(J_{A}\right)=0.6770$.

\section{Competing interests}

The authors declare that they have no competing interests.

\section{Authors' contributions}

All authors conceived of the study, participated in its design and coordination, drafted the manuscript, participated in the sequence alignment, and read and approved the final manuscript.

\section{Acknowledgements}

This research is supported by the National Natural Science Foundations of China (No. 10802068).

Received: 29 November 2014 Accepted: 16 February 2015 Published online: 04 March 2015

\section{References}

1. Xu, Z, Lu, Q, Zhang, KY, An, XH: Theory and Application of H-Matrices. Science Press, Beijing (2013)

2. Huang, R: Some inequalities for the Hadamard product and the fan product of matrices. Linear Algebra Appl. 428 1551-1559 (2008)

3. Liu, QB, Chen, GL, Zhao, LL: Some new bounds on the spectral radius of matrices. Linear Algebra Appl. 432, 936-948 (2010)

4. Kolotilina, LY: Bounds for the Perron root, singularity/nonsingularity conditions, and eigenvalue inclusion sets. Numer. Algorithms 42, 247-280 (2006)

5. Yong, XR, Wang, Z: On a conjecture of Fiedler and Markham. Linear Algebra Appl. 288, 259-267 (1999)

6. Cheng, GH, Cheng, XY, Huang, TZ, Tam, TY: Some bounds for the spectral radius of the Hadamard product of matrices. Appl. Math. E-Notes 5, 202-209 (2005)

7. Brauer, A: Limits for the characteristic roots of a matrix. Duke Math. J. 14, 21-26 (1947)

8. Varga, RS: Minimal Gerschgorin sets. Pac. J. Math. 15(2), 719-729 (1965)

9. Guo, QP, Li, HB, Song, MY: New inequalities on eigenvalues of the Hadamard product and the fan product of matrices. J. Inequal. Appl. 2013, 433 (2013) 
10. Liu, QB, Chen, GL: On two inequalities for the Hadamard product and the fan product of matrices. Linear Algebra Appl. 431, 974-984 (2009)

11. Fang, MZ: Bounds on eigenvalues of the Hadamard product and the fan product of matrices. Linear Algebra Appl. $425,7-15(2007)$

Submit your manuscript to a SpringerOpen ${ }^{\circ}$ journal and benefit from:

- Convenient online submission

Rigorous peer review

- Immediate publication on acceptance

Open access: articles freely available online

- High visibility within the field

- Retaining the copyright to your article 